\title{
A nomogram for clinical estimation of acute biliary pancreatitis risk among patients with gallstones
}

Xiaoyu Guo

Harbin Medical University

Yilong Li

Harbin Medical University

Hui Lin

Shanxi Medical University

Jie Li

Harbin Medical University

Long Cheng

Harbin Medical University

Zijian Huang

Harbin Medical University

Zhitao Lin

Harbin Medical University

Ning Mao

Harbin Medical University

Linfeng Wu

Harbin Medical University

Bei Sun

Harbin Medical University

Weidong Wu

Shanxi Medical University

Gang Wang ( $\square$ wgilu79@163.com )

Harbin Medical University

\section{Research}

Keywords: Gallstones, acute biliary pancreatitis, predictors, nomogram, receiver operating characteristic curves

Posted Date: April 7th, 2021

DOI: https://doi.org/10.21203/rs.3.rs-377101/v1 
License: (c) (i) This work is licensed under a Creative Commons Attribution 4.0 International License. Read Full License 


\section{Abstract}

Background Currently, there are no effective tools to accurately assess acute biliary pancreatitis (ABP) risk in patients with gallstones. This study aimed to develop an ABP risk nomogram in patients with gallstones.

Methods Data on 2102 patients with gallstones admitted to the Department of General Surgery of The First Affiliated Hospital of Harbin Medical University between January 6, 2009 and January 22, 2018 were retrospectively collected. Some patients were randomly divided into the training cohort $(n=1470)$ for nomogram development; the others formed the validation cohort $(n=632)$ to confirm the model's performance. The chi-square test was used to optimize feature selection, and logistic regression analysis was applied to build a prediction model incorporating the selected features. The area under the curve (AUC), Hosmer-Lemeshow test, calibration curve, decision curve analysis (DCA) and internal validation were used to validate the model's accuracy, calibration and clinical effectiveness.

Results Predictors included sex (male), diabetes, gallbladder wall thickness $\leq 3 \mathrm{~mm}$, gallbladder stone diameter $<3 \mathrm{~mm}$, coexisting CBD stone, CBD diameter $\leq 10 \mathrm{~mm}, \mathrm{AST} \geq 53.6 \mathrm{U} / \mathrm{L}, \mathrm{GGT} \geq 150 \mathrm{U} / \mathrm{L}, \mathrm{DBIL} \geq 1.0$ $\mathrm{mg} / \mathrm{dL}, \mathrm{WBC} \geq 1010^{9} 0^{\circ}$, and GRAN\% $\geq 80 \%$. The model displayed good predictive power with AUCs of 0.850 (95\% Cl: $0.825 ~ 0.875)$ and 0.844 (95\% Cl: $0.825 ~ 0.875)$ in the training and validation cohorts, respectively. The DCA showed a $10-100 \%$ risk threshold. The Hosmer-Lemeshow test and calibration curve demonstrated the accuracy and effectiveness of this model, which could be applied in clinical practice.

Conclusions The ABP risk nomogram incorporating 11 features is useful to predict ABP risk in gallstone patients.

\section{Introduction}

Acute pancreatitis (AP), as a common acute abdominal condition, is an inflammatory disorder of the pancreas accompanied by potentially severe local or systemic complications and high mortality[1]. As a leading cause of admission to the hospital for gastrointestinal disorders worldwide, AP is characterized by the main clinical feature of autodigestion of the pancreas, sometimes accompanied by multiple organ dysfunction[2]. There are various etiological factors capable of inducing an acute attack of AP, such as gallstones, alcohol misuse, smoking, drug use, genetic factors, and tumors[3, 4]. According to these factors, AP is frequently divided into acute biliary pancreatitis (ABP), acute alcoholic pancreatitis, acute hyperlipidemic pancreatitis, acute idiopathic pancreatitis and so forth[5].

ABP is recognized as the leading type of AP worldwide, accounting for $35-60 \%$ of AP cases, with a reported mortality rate ranging from $5 \%$ to $20 \%$.[6] The pathogenesis of $A B P$ might be associated with passage of small gallbladder stones or biliary sludge through the ampulla of Vater and other factors, such as anatomical variations, iatrogenic factors including surgical operation and endoscopic retrograde cholangiopancreatography (ERCP), ampullary carcinoma, and pancreatic head carcinoma[6, 7]. Of all 
these risk factors, gallstones are still the main cause of ABP. Studies suggest that ABP is frequently the first symptom of gallstone disease in approximately $40 \%$ of patients without a preceding episode of biliary colic[8]. Clinical data and experience also show that not all patients with gallstones will eventually develop ABP, which results in an arduous challenge and several questions: Which patients with gallstones are more likely to develop ABP? What are the related risk factors for ABP? How can clinicians accurately predict the occurrence of $\mathrm{ABP}$ in patients with gallstones and take timely preventive measures?

Owing to the lack of a specific and effective predictive technique, the development of a prediction model that incorporates distinctive ABP-related factors based on clinicopathologic data is desirable. Of all the available models, a nomogram can provide an individualized, evidence-based, highly accurate risk estimation. Nomograms are easy to use and can facilitate management-related decision making[9]. To our knowledge, we have established the first nomogram for ABP risk among patients with gallstones.

\section{Methods}

\section{Patients}

Between January 6, 2009, and January 22, 2018, data on inpatients who had been diagnosed with gallstones were retrospectively collected from the First Affiliated Hospital of Harbin Medical University (Figure 1). All patients assigned to the ABP group were confirmed to be diagnosed with ABP at a later time. The study was approved by the Institutional Ethics Committee of the hospital. All the patients' data were used for only research. The study did not affect the treatment of patients.

The inclusion criteria were mainly related to the confirmation of gallstones using ultrasound, computed tomography (CT), magnetic resonance cholangiopancreatography (MRCP) or other imaging examinations. The exclusion criteria included (1) incomplete medical record data and serum test and imaging examination results, (2) AP induced by other factors, such as acute hyperlipidemic pancreatitis; acute hypercalcemia pancreatitis; and AP caused by anatomic abnormality of pancreas, pancreatic cancer, drugs, overeating, alcohol misuse, operation (including ERCP), severe injury, infection, and biliary tract ascariasis, (3) ambiguous diagnosis, (4) other concomitant major diseases that would interfere with the study, such as heart failure, renal failure, and multiple organ dysfunction syndrome (MODS), and (5) female AP patients during pregnancy or lactation.

\section{ABP diagnostic criteria}

The ABP diagnostic criteria were as follows: (1) having gallstones confirmed by abdominal ultrasound, $\mathrm{CT}, \mathrm{MRCP}$ or other imaging examination; (2) having two or more of the following laboratory examination indicators: 『alkaline phosphatase (AKP) $>125 \mathrm{U} / \mathrm{L}$, 区alanine transaminase (ALT) $>150 \mathrm{U} / \mathrm{L}$, \total bilirubin (TBIL) $>2.3 \mathrm{mg} / \mathrm{dl}$, and $\llbracket$ gamma-glutamyl transferase (GGT)>40 U/L; (3) conforming to the diagnostic

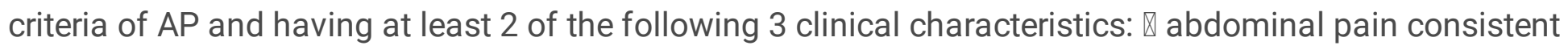

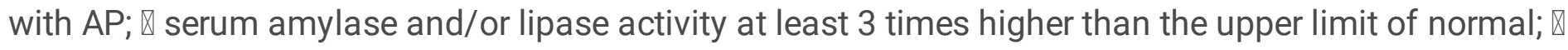


abdominal imaging examination consistent with the imaging changes associated with AP; and (4) no other causes of abnormality of serum amylase and lipase and liver function test[10-13].

\section{Clinicopathologic variables}

The clinicopathologic variables in this study are reported in Table 1, and these variables were recorded before diagnosis of ABP. The imaging data included gallbladder size, thickness of the gallbladder wall, number of gallstones, diameter of gallstones, gallstone morphology, coexisting CBD stone, and diameter of the bile duct. The normal size of the adult gallbladder was defined as $7-10 \mathrm{~cm}$ in length and $3-4 \mathrm{~cm}$ in transverse diameter[14]. A gallbladder with a size differing from this criteria was seen as abnormal. The gallbladder wall thickness, as an objective marker of cholecystitis severity and laparoscopic cholecystectomy (LC) complexity, was divided into two groups: $\leq 3 \mathrm{~mm}$ (normal) and $>3 \mathrm{~mm}[15]$. The characteristics of gallstones were represented by the number of gallstones, the diameter of gallstones and the gallstone shapes. The number of gallstones was divided into two groups: solitary gallstones and multiple gallstones $(\geq 2)$. According to the gallstone shapes shown by imaging examinations, the shapes were classified into spherical stones, sand-like stones and irregular stones. Considering that smaller gallstones were frequently seen in patients with $\mathrm{AP}$, especially stone sizes $<3 \mathrm{~mm}$, the diameter of gallstones was separated into three groups: $<3 \mathrm{~mm}, 3 \sim 10 \mathrm{~mm}$ and $>10 \mathrm{~mm}$, and the minimum diameter of gallstones was recorded in case of multiple gallstones[16]. During the imaging examination, two experienced radiologists independently evaluated all imaging data. Any controversies in imaging findings between radiologists were settled by discussion, and a final standard radiologic report on each patient was generated. At present, although abdominal ultrasound is still the most common method of screening for gallstones and bile duct stones, due to intestinal gas interference, the diagnostic value is always limited[17]. CT is clinically more valuable than ultrasound in diagnosing gallstones and bile duct stones, but it is easily affected by stone composition and density, especially low-density stones with cholesterol as the main component[18]. In contrast, MRCP's sensitivity, specificity, and diagnostic accordance rate in the diagnosis of gallstones are higher than those of ultrasound and CT[19]. Therefore, the imaging results of MRCP were recorded preferentially in this study.

\section{Statistical analysis}

We analyzed the differences in the distribution of all clinical baseline characteristics in the ABP group in the training cohort and described them by using the number of cases. We performed a chi-square test and multivariate logistic regression analysis with results presented as odds ratios (ORs) with $95 \%$ confidence intervals (Cls). In the multivariate regression analysis, we used stepwise backward elimination and set the level of significance to $P<0.05$. We drew receiver operating characteristic $(R O C)$ curves to evaluate the predictive value of the established multivariate logistic regression model and employed the HosmerLemeshow test and calibration curve to further evaluate the accuracy of the model. Decision curve analysis (DCA) was also utilized to evaluate the clinical application value of the model. Moreover, for the clinical use of the model, we drew a nomogram based on a multivariate logistic regression model and ensured that the total scores of each patient could be effectively calculated by this nomogram. All 
analyses were performed using R, version 3.6.3. Data analysis was conducted from November 6, 2019 to February 3, 2020.

\section{Results}

\section{Clinicopathologic characteristics and univariate analysis results}

In this study, after exclusion, 2102 patients with gallstones who met the inclusion criteria were finally enrolled, and 1470 and 632 patients were divided into the training and validation cohorts, respectively (Figure 1). Of these, a total of 488 patients with gallstones had been diagnosed with ABP at the time the study began.

The clinicopathologic characteristics of the patients are listed in Table 1. The baseline clinicopathologic data were similar between the training and validation cohorts. Clinically identified ABP was found in 341 $(23.2 \%)$ and 147 (23.3\%) patients in the training and validation cohorts, respectively. The univariate analysis results of the training cohort showed that sex, smoking history, diabetes history, gallbladder wall thickness, gallstone number, gallstone diameter, gallstone shape, coexisting CBD stone, ALT, aspartate transaminase (AST), GGT, AKP, TBIL, indirect bilirubin (IBIL), direct bilirubin (DBIL), white blood cell count (WBC) and granulocyte\% (GRAN\%) were significantly different between the ABP group and the non-ABP group $(p<0.05)$.

\section{Multivariate analysis result and establishment of an ABP-predicting nomogram}

All variables used in this analysis were based on the data obtained retrospectively. The gallbladderrelated variables, including gallbladder size, gallbladder wall thickness, gallstone diameter, gallstone shape, coexisting CBD stone and diameter of the common bile duct (CBD), were assessed by imaging studies.

The significant factors obtained in the univariate analysis results were included in the multivariate logistic regression model to analyze whether each factor was an independent risk factor for inducing ABP. Additionally, according to clinical experience, gallbladder size and CBD diameter were included in this model as covariables to be calibrated. After screening independent variables by means of gradual screening, we obtained multivariate logistic regression analysis results (Table 2).

In the multivariate analysis, with results reported as ORs (95\% Cls), male sex (1.940 [1.436-2.628]), diabetes history (2.279 [1.657-3.132]), gallbladder wall thickness $>3 \mathrm{~mm}(0.633$ [0.452-0.882]), gallstone diameter (3-10 vs $<3 \mathrm{~mm}, 0.489$ [0.343-0.697], $>10 \mathrm{~mm}$ vs $<3 \mathrm{~mm}, 0.489$ [0.343-0.697]), coexisting CBD stone (2.062 [1.366,3.115]), CBD diameter >10 mm (0.507 [0.296-0.853]), AST >53.6 U/L (2.013 [1.362,2.974]), GGT>150 U/L (2.306 [1.574-3.380]), DBIL<1.0 mg/dL (0.263 [0.127-0.549]), WBC>10í109 (4.201 [3.073-5.767]), and GRAN\%>80\% (2.997 [2.211-4.071]) were independently associated with ABP (Table 2, Figure 2). These independently associated risk factors were utilized to make an ABP risk estimation nomogram (Figure 3). 


\section{Validation and effect evaluation of the nomogram}

The resulting model was internally validated using the bootstrap validation method. The performance of the nomogram was measured by ROC curves (Figure 4). In the training cohort, the cut-off score was 0.323 with a sensitivity of $72.4 \%$ and a specificity of $86.0 \%$, and the maximum Youden index was obtained. The AUC was 0.850 (95\% $\mathrm{Cl} 0.825-0.875)$. In addition, calibration plots graphically showed good agreement in regard to the presence of $A B P$ between the risk estimation by the nomogram and clinical confirmation (Figure 5). Moreover, in the validation cohort, the resulting model displayed an AUC of $0.844(95 \% \mathrm{Cl}$ 0.808-0.880) for the estimation of ABP risk (Figure 4). The Hosmer-Lemeshow test was further used to test the fitting accuracy of the logistic regression model. When groups were $5, \chi^{2}=4.072$, the degree of freedom $=3$, and $P=0.254$. When groups were $100, \chi^{2}=106.66$, the degree of freedom $=98$, and $P=0.258$. This result indicated that the prediction model had good accuracy. The decision curve analysis for the ABP incidence risk nomogram is presented in Figure 6. The decision curve demonstrated good clinical value of the nomogram, and when the value of threshold probability (Pt) was set between $10 \%$ and $100 \%$, there was a net benefit of using this $A B P$ nomogram to predict $A B P$ risk.

\section{Discussion}

AP is characterized by acute onset, rapid progression and a high likelihood of developing severe acute pancreatitis (SAP), with severe complications and a high mortality rate of $30 \%[1]$. Gallstones are still one of the key causative factors of AP, and in the present study, approximately $20 \%$ of gallstone patients in hospitals were diagnosed with $\mathrm{ABP}[13]$. Therefore, it is clinically important for clinicians to prevent gallstone patients from developing AP and SAP[20]. Our study also suggests that risk factors, including male sex, diabetes history, gallbladder wall thickness less than $3 \mathrm{~mm}$, gallstone diameter less than $3 \mathrm{~mm}$, coexisting CBD stone, CBD diameter less than $10 \mathrm{~mm}$, AST greater than $53.6 \mathrm{U} / \mathrm{L}, \mathrm{GGT}$ greater than 150 $\mathrm{U} / \mathrm{L}$, DBIL greater than $1.0 \mathrm{mg} / \mathrm{dL}, \mathrm{WBC}$ greater than $1010^{\circ}$, and GRAN\% greater than $80 \%$, are significantly associated with the incidence of $A B P$.

Previous studies showed that gallstones were more commonly associated with AP in women[21, 22]. However, in this study, multivariate analysis results indicated that male sex was a risk factor for ABP, and the risk among men was 1.940-times higher than that among women. On the one hand, as noted earlier, female patients with gallstones were more likely to undergo cholecystectomy than male patients[23]. On the other hand, this result might be related to the local people's lifestyles: men are more likely to drink heavily and consume fatty food. In addition, diabetes history is another important risk factor for ABP (OR: 2.279ष95\% Cl: 1.657 3.132). Relevant studies have demonstrated that although some controversy exists, diabetic patients are generally thought to have a twofold to threefold increased risk of cholesterol gallstones[24]. Moreover, owing to poor anti-infection ability and immunity, when patients with diabetes suffer cholecystitis, they are prone to serious biliary tract infection and even other severe complications, such as gallbladder abscess, gangrene, and perforation[25]. We speculate that diabetes is more likely to be associated with biliary tract infection and that biliary tract function is worse in patients with diabetes 
than in the normal population. Therefore, gallstone incarceration is more likely to occur during the downward movement of gallstones, thus inducing ABP.

Among the factors related to gallstones and the biliary tract, this study showed that the thickness of the gallbladder wall, gallstone diameter, CBD diameter and coexisting CBD stone were all significant risk factors for $A B P$ in patients with gallstones. The risk of $A B P$ in the normal gallbladder group was significantly higher than that in the abnormal gallbladder group. In addition, gallbladder wall thickness $\leq$ $3 \mathrm{~mm}$ was a risk factor for $A B P$ (in comparison, the OR of the thickness of $>3 \mathrm{~mm}$ was $0.633,95 \% \mathrm{Cl}$ : $0.452 \sim 0.882)$; that is, the risk of $A B P$ in patients with a thickness of the gallbladder wall $\leq 3 \mathrm{~mm}$ was 1.58 times as high as that of patients with a thickness of $>3 \mathrm{~mm}$. The reason might be that when the gallbladder wall thickness is normal, the gallbladder's contraction function is relatively good[26]. Therefore, when the gallbladder contracts, the gallbladder tube is able to normally expand, thus making gallstones easily discharged into the CBD and inducing ABP. In contrast, long-term inflammation frequently leads to a thicker gallbladder wall and relatively poor gallbladder contraction function, and the gallbladder duct has difficulty expanding[27]. Therefore, gallstones are difficult to discharge and are likely to remain in the gallbladder, making the risk of ABP relatively lower. In the gallstone size analysis, patients were divided into three groups according to stone diameter: $<3 \mathrm{~mm}, 3-10 \mathrm{~mm}$ and $>10 \mathrm{~mm}$, and patients with gallstone diameters $<3 \mathrm{~mm}$ were 2.04 times more likely to develop ABP than those with diameters $3-10 \mathrm{~mm}$ and 2.71 times more likely to develop ABP than those with diameters $>10 \mathrm{~mm}$. The reason may be that when the gallbladder contracts, stones with smaller diameters are more likely to enter the common channel, and they easily cause channel blockage and finally induce ABP. In contrast, larger diameter stones tend to be incarcerated in the gallbladder neck and do not easily enter the common channel. Therefore, the risk of inducing ABP is relatively reduced[6, 28]. Coexisting CBD stone were also an essential risk factor for ABP in gallstone patients (OR: 2.062, 95\% Cl: 1.366 3.115). Our analysis showed that the risk of $A B P$ in patients with gallstones and bile duct stones was twice as high as that in patients with only gallstones. The reason is possibly that compared with gallstones, bile duct stones are more likely to cause duodenal papillary edema or stenosis, especially in the ampullary segment and the lower CBD, which are more likely to result in ABP than the upper CBD[29]. Previous studies have shown that a dilated CBD was one of the pathogenic factors of AP, possibly because gallstones were more likely to pass through the relatively dilated CBD, and the risk of causing duodenal papillary edema or stenosis was reasonably higher[6]. Although the diameter of the CBD was not statistically significant in the univariate analysis, we still included it as a covariate to be corrected in the multivariate analysis. The results demonstrated that a diameter of the $C B D \leq 10$ was a risk factor for $A B P$, and the risk of $A B P$ in patients with gallstones and a normal bile duct diameter was 1.97 times higher than that in patients with a bile duct diameter $>10 \mathrm{~mm}$. In our experience, small granular stones in the gallbladder are often excreted through the duodenal papillary sphincter, and their temporary retention always gives rise to duodenal papillary edema and transient biliary obstruction, thus leading to bile reflux and acute biliary pancreatitis[10]. It is exactly because of the transient stone slip and short-term block that most of the ultrasound and CT examinations have difficulty providing obvious evidence of bile duct obstruction or dilation, which is also consistent with the "common channel theory"[30]. 
In terms of biochemical indicators commonly used in clinical practice, combined with multivariate logistic regression analysis, we demonstrated that AST, GGT, DBIL, and GRAN\% were all important and independent risk factors for $\mathrm{ABP}$ development in patients with gallstones. Among these risk factors, the abnormal levels of AST, GGT and DBIL reflect the degree of bile duct obstruction during the pathogenesis of $A B P$ and the degree of hepatocyte injury caused by bile reflux[12]. Moreover, the levels of WBC and GRAN\% are able to reflect the severity of biliary tract infection during ABP[2]. In general, when patients with gallstones suffer severe obstruction and infection of the biliary tract, their risk of concurrent ABP is obviously higher, and these biochemical indicators have good prediction value for ABP.

Previous studies mostly focused on the important risk factors in the analysis of the results, and they were not able to clearly calculate the risk of ABP in every patient with gallstones. In addition, the application of the traditional logit formula had to rely on computers and software and was difficult to apply on portable devices. Of the currently available prediction tools, a nomogram has high accuracy and good discrimination characteristics in predicting outcomes, and it is easy to use[31]. In the present study, the proposed nomogram, which incorporated 11 comprehensive and easily available clinical variables, performed well and was supported by AUCs of 0.850 and 0.844 in the training and validation cohorts, respectively, and the optimal calibration curves demonstrated agreement between the prediction and actual observation. In the process of evaluating the effectiveness of statistical models, traditional methods such as ROC curves cannot prevent false positives and false negatives. Therefore, to improve the accuracy of clinical decision making, DCA was introduced to further verify the clinical effectiveness of the prediction model. In the decision curve, when the Pt value was set between $10 \%$ and $100 \%$, the curve was significantly higher than the two extreme curves; that is, patients could gain positive net benefits. In other words, in this study, the decision curve established according to the prediction model was basically above the two extreme curves (when $<10 \%$, the curve almost overlapped with the extreme curve of all interventions), which further verified the predictive value and application value of the prediction model and could be applied to actual clinical decision making[32].

There are no international guidelines that indicate which patient to offer a cholecystectomy or conservative treatment. Therefore, the indication to perform a cholecystectomy always lies within the surgeons' preference leading to variations in practice and consequently unnecessary cholecystectomies[33]. Some findings show that asymptomatic gallstone patients should not undergo prophylactic cholecystectomy. In a study by Gracie et al., only $18 \%$ of asymptomatic gallstone carriers developed biliary pain or a gallstone complication during 15-yr follow-up[34]. Furthermore, symptomatic complicated gallstone patients, especially ones with mild-to-moderate acute cholecystitis, common bile duct stones, or mild biliary pancreatitis, are now recommended for same admission cholecystectomy as opposed to delayed cholecystectomy in previous guidelines[35].

However, for symptomatic uncomplicated gallstone patients, who are primarily involved in this study, whether and when cholecystectomy should be performed remains controversial. Most studies recommend that cholecystectomy is the therapy of the first choice for patients with uncomplicated symptomatic gallstone disease. Many patients have had an unnecessary cholecystectomy with 
associated risks of complications and unnecessary healthcare expenses, and some studies indicated that up to $33 \%$ of patients do not experience relief of their abdominal symptoms, despite cholecystectomy[36]. Moreover, some patients with suggested uncomplicated symptomatic gallstone disease should be treated conservatively because of a high risk of persistent symptoms or suboptimal benefit of cholecystectomy. However, from the perspective of ABP prevention, there are still no uniform guidelines for choosing the optimal timing of cholecystectomy. Although some patients are at high risk of ABP in clinical practice, they may still choose conservative treatment and refuse effective surgical treatment for some reasons. All these above may increase the potential risk of ABP in patients with gallstones. In this study, most of the samples are asymptomatic uncomplicated patients with gallstones. Therefore, the nomogram can be used to identify these patients' potential risk for ABP through commonly used clinical indicators and to help clinicians make better clinical decisions on the optimal timing of cholecystectomy. Moreover, it is also beneficial to encourage patients to avoid risk factors for ABP and receive a medical check-up regularly. Finally, it is worth noting that in this study, decision curves indicate that when the risk of $A B P$ in patients is greater than $10 \%$, carrying effective intervention will bring the population positive overall benefit. However, the intervention may include regular follow-up, regular medical check-ups, endoscopic treatment and surgical treatment. According to patients' different risks for ABP, which intervention effectively prevents the occurrence of ABP remains to be further studied.

Our study had some limitations. First, this analysis was based on data from a single institution; it is necessary to validate the results in other centers. Second, there are still many risk factors affecting the incidence of $A B P$ in patients with gallstones. Due to limited data, the risk factors selected in this study were not complete. Some potential risk factors of patients, such as body mass index (BMI) and blood lipids, were not included in the study, and more risk factors should be included in this study to further improve the accuracy of the prediction model. Finally, although the nomogram is more convenient than the traditional statistical model, it is no denying that there are still some limitations in the actual application. In the future, we will put the scoring system on a website or an app for use on a smart phone for surgeons in the hospital, and the score could automatically calculate results online.

\section{Conclusion}

By combining 11 clinical risk factors for ABP in gallstone patients, a nomogram was constructed. The model provides an accurate and optimal estimation of ABP risk in patients with gallstones. The nomogram provides an effective tool for quantitative clinical assessment of risks and benefits, which is conducive to the early prevention and treatment of $A B P$ in patients with gallstones. This model could also help clinicians and patients make scientific clinical decisions to maximize the clinical benefits of patients.

\section{Abbreviations}

ABP: Acute biliary pancreatitis; AKP: Alkaline phosphatase; ALT: Alanine transaminase; AP: Acute pancreatitis; AST: Aspartate transaminase; AUC: Area under the curve; BMI: Body mass index; CBD: 
Common bile duct; Cis: Confidence intervals: CT: Computed tomography; DBIL: Direct bilirubin; DCA: Decision curve analysis; ERCP: Endoscopic retrograde cholangiopancreatography; GGT: Gamma-glutamyl transferase; GRAN\%: Granulocyte\%; IBIL: Indirect bilirubin; LC: Laparoscopic cholecystectomy; MODS: Multiple organ dysfunction syndrome; MRCP: Magnetic resonance cholangiopancreatography; ORs: Odds ratios; Pt: Threshold probability; ROC: Receiver operating characteristic; SAP: Severe acute pancreatitis; TBIL: Total bilirubin; WBC: White blood cell count.

\section{Declarations}

\section{Acknowledgement}

none.

\section{Authors' contributions}

$X G, H L$, and $Y L$ contributed equally to the study initial design, analysis, interpretation of data, drafting of the initial manuscript, critical revision of intellectual content. JL, LC, ZH, ZL, and NM participated in the data collection and statistical analysis. LW, BS, WW, and GW contributed to the study design and coordination and helped to draft the manuscript. All authors read and approved the final manuscript.

\section{Funding}

This paper was supported by grants from the National Nature Scientific Foundation of China ه81770639,82070657囚, Applied technology research and development project of Heilongjiang Province \GA20C019ه, Outstanding youth funds of the first affiliated hospital of Harbin Medical University

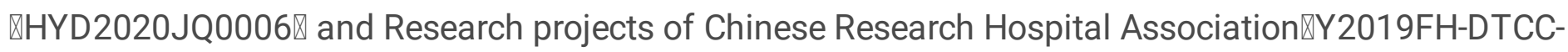
SB1区.

\section{Availability of data and materials}

The datasets generated and analyzed during the current study are available from the corresponding author on reasonable request.

\section{Ethics approval and consent to participate}

The study was approved by the Institutional Ethics Committee of the hospital. Patients' consent are waived.

\section{Consent for publication}

Not applicable.

\section{Competing interests}

The authors declare that they have no competing interests. 


\section{References}

1. Guo XY, Xiao F, Li J et al. Exosomes and pancreatic diseases: status, challenges, and hopes. Int J Biol Sci. 2019;15:1846-1860.

2. Lankisch PG, Apte PM, Banks PA. Seminar Acute pancreatitis. The Lancet 2015;386:1-12.

3. Lowenfels $A B$, Lankisch PG, Maisonneuve P. What is the risk of biliary pancreatitis in patients with gallstones? Gastroenterology. 2000;119:879-80.

4. Lankisch PG, Lowenfels $A B$, Maisonneuve $P$. What is the risk of alcoholic pancreatitis in heavy drinkers? Pancreas. 2002;25:411-12.

5. Nauck MA. A critical analysis of the clinical use of incretin-based therapies: the benefits by far outweigh the potential risks. Diabetes Care. 2013,36:2126-32.

6. van Geenen EJ, van der Peet DL, Bhagirath P, et al. Etiology and diagnosis of acute biliary pancreatitis. Nat Rev Gastroenterol Hepatol. 2010;7(9):495-502.

7. Ridtitid W, Kulpatcharapong S, Piyachaturawat P, et al. The impact of empiric endoscopic biliary sphincterotomy on future gallstone-related complications in patients with non-severe acute biliary pancreatitis whose cholecystectomy was deferred or not performed. Surg Endosc. 2019;33: 33253333.

8. van Erpecum KJ. Gallstone disease. Complications of bile-duct stones: acute cholangitis and pancreatitis. Best Pract Res Clin Gastroenterol. 2006;20:1139-1152.

9. Balachandran VP, Gonen M, Smith JJ, et al. Nomograms in oncology: more than meets the eye. Lancet Oncol. 2015;16:e173-80.

10. Zhao YP. Diagnostic criteria and treatment principles of billary pancreatitis. Chin J Hepatobiliary Surg. 2002;8(2):95-96.

11. Coffey MJ, Nightingale S, Ooi CY. Predicting a biliary aetiology in paediatric acute pancreatitis. Arch Dis Child. 2013;98(12):965-969.

12. Surlin V, Saftoiu A, Dumitrescu D. Imaging tests for accurate diagnosis of acute biliary pancreatitis. World J Gastroenterol. 2014;20(44):16544-16549.

13. Zhu SL, Lin QT. Diagnosis and treatment of biliary pancreatitis. J Hepatopancreatobiliary Surg. 2012; 24(1):38-40.

14. John RG, Laura WL, Jesse M, et al. Rosai and Ackerman's Surgical Pathology; 2017.

15. Kokoroskos N, Peponis T, Lee JM, et al. Gallbladder wall thickness as a predictor of intraoperative events during laparoscopic cholecystectomy: A prospective study of 1089 patients. Am J Surg. 2020;Mar(08). doi:10.1016/j.amjsurg.2020.03.007.

16. Gonzalez RR, Garza AG, Martinez L, et al. Evaluation of previous abdominal pain episodes in women with biliary pancreatitis: possible higher incidence of recurrent attacks. Tropical Gastroenterology. 2012;33:270-274. 
17. Soyer P, Hoeffel C, Dohan A, et al. Acute cholecystitis: quantitative and qualitative evaluation with 64section helical CT. Acta Radiol. 2013;54(5):477-486.

18. Chan WC, Joe BN, Coakley FV, et al. Gallstone detection at CT in vitro: effect of peak voltage setting. Radiology. 2006;241(2):546-553.

19. Griffin N, Charles-Edwards G, Grant LA. Magnetic resonance cholangiopancreatography: the $A B C$ of MRCP. Insights Imaging. 2012;3(1):11-21.

20. Lyu YX, Cheng YX, Jin HF, et al. Same-admission versus delayed cholecystectomy for mild acute biliary pancreatitis: a systematic review and meta-analysis. BMC Surg. 2018;18:111.

21. Setiawan VW, Monroe K, Lugea A, et al. Uniting Epidemiology and Experimental Disease Models for Alcohol-Related Pancreatic Disease. Alcohol Res. 2017;38:173-82.

22. Yadav D, Lowenfels AB. The epidemiology of pancreatitis and pancreatic cancer. Gastroenterology. 2013;144:1252-61.

23. Locke GR. Digestive diseases in the United States: Epidemiology and impact. National Institutes of Health. 1995;108:946-946. .

24. Chen $\mathrm{CH}$, Cheng LL, Hsu CY, et al. Association Between Type I and II Diabetes With Gallbladder Stone Disease. Frontiers in Endocrinology. 2018;9(720). doi: 10.3389/fendo.2018.00720.

25. Ransohoff DF, Miller GL, Forsythe SB, et al. Outcome of acute cholecystitis in patients with diabetes mellitus. Ann Intern Med. 1987;106:829-32.

26. Yamada K, Yamada H. Gallbladder wall thickening in mononucleosis syndromes. J Clin Ultrasound. 2001;29(6):322-5.

27. Stads $S$, Venneman NG, Scheffer RC, et al. Evaluation of gallbladder motility: comparison of twodimensional and three-dimensional ultrasonnography. Ann Hepatol. 2007;6(3):164-169.

28. Kim SB, Kim TN, Chung HH, et al. Small Gallstone Size and Delayed Cholecystectomy Increase the Risk of Recurrent Pancreatobiliary Complications After Resolved Acute Biliary Pancreatitis. Dig Dis Sci. 2017;62:777-783.

29. Lee SL, Kim HK, Choi HH, et al. Diagnostic value of magnetic resonance cholangiopancreatography to detect bile duct stones in acute biliary pancreatitis. Pancreatology. 2018;18:22-28.

30. Siqin D, Wang $C$, Zhou $Z$, et al. The key event of acute pancreatitis: pancreatic duct obstruction and bile reflux, not a single one can be omitted. Med Hypotheses. 2009;72(5):589-591.

31. Shariat SF, Capitanio U, Jeldres $C$, et al. Can nomograms be superior to other prediction tools? BJU Int. 2009;103(4):492-495.

32. Vickers AJ, Cronin AM, Elkin EB, et al. Extensions to decision curve analysis, a novel method for evaluating diagnostic tests, prediction models and molecular markers. BMC Med Inform Decis Mak. 2008;8:53.

33. Harrison EM, O'Neill S, Meurs TS, et al. Hospital volume and patient outcomes after cholecystectomy in Scotland: retrospective, national population based study. BMJ. 2012; 344:e3330. 
34. Gracie WA, Ransohoff DF. The natural history of silent gallstones: the innocent gallstone is not a myth. N Engl J Med. 1982; 307:798-800.

35. Lamberts MP. Indications of cholecystectomy in gallstone disease. Curr Opin Gastroenterol. 2018; 34 : 97-102.

36. Lamberts MP, Lugtenberg M, Rovers MM, et al. Persistent and de novo symptoms after cholecystectomy: a systematic review of cholecystectomy effectiveness. Surg Endosc. 2013; 27:709-718.

\section{Tables}

Table-1. Clinical baseline characteristics of patients included and univariate analysis results of training set 


\begin{tabular}{|c|c|c|c|c|c|}
\hline \multirow[t]{3}{*}{ Variable } & \multicolumn{4}{|c|}{ Training cohort $[n=1470 \square$} & \multirow{3}{*}{$\begin{array}{l}\text { Validation } \\
\text { cohort } \\
\text { [n=632] }\end{array}$} \\
\hline & ABP & Non-ABP & $x^{2}$ & $P$ & \\
\hline & $\mathrm{n} \otimes \% \bigotimes$ & $\mathrm{n} \otimes \% \bigotimes$ & & & \\
\hline Age $\bigotimes$ year》 & & & 2.565 & 0.109 & \\
\hline$\nabla 50$ & $469(41.54 \%)$ & $159(46.63 \%)$ & & & $271(42.88 \%)$ \\
\hline$\geq 50$ & $660(58.46 \%)$ & $182(53.37 \%)$ & & & $361(57.12 \%)$ \\
\hline Sex & & & 31.744 & $<0.001^{*}$ & \\
\hline Female & $671(59.43 \%)$ & $143(41.94 \%)$ & & & $323(51.11 \%)$ \\
\hline Male & $458(40.57 \%)$ & $198(58.06 \%)$ & & & $309(48.89 \%)$ \\
\hline Alcoholic history & & & 10.455 & $0.001^{*}$ & \\
\hline No & $935(82.82 \%)$ & $255(74.78 \%)$ & & & $502(79.43 \%)$ \\
\hline Yes & 194(17.18\%) & $86(25.22 \%)$ & & & $130(20.57 \%)$ \\
\hline Smoking history & & & 8.344 & $0.004^{*}$ & \\
\hline No & $925(82.00 \%)$ & $254(74.71 \%)$ & & & $517(81.8 \%)$ \\
\hline Yes & $203(18.00 \%)$ & $86(25.29 \%)$ & & & $115(18.2 \%)$ \\
\hline Diabetes history & & & 77.448 & $<0.001^{*}$ & \\
\hline No & $905(80.16 \%)$ & $192(56.30 \%)$ & & & $471(74.53 \%)$ \\
\hline Yes & $224(19.84 \%)$ & $149(43.70 \%)$ & & & $161(25.47 \%)$ \\
\hline Gallbladder size & & & 2.21 & 0.137 & \\
\hline Normal & $778(68.91 \%)$ & $250(73.31 \%)$ & & & $445(70.41 \%)$ \\
\hline Abnormal & $351(31.09 \%)$ & $91(26.69 \%)$ & & & $187(29.59 \%)$ \\
\hline $\begin{array}{l}\text { Gallbladder wall thickness } \\
(\mathrm{mm})\end{array}$ & & & 4.491 & $0.034^{*}$ & \\
\hline$\leq 3$ & $736(65.19 \%)$ & $244(71.55 \%)$ & & & $401(63.45 \%)$ \\
\hline$凶 3$ & $393(34.81 \%)$ & $97(28.45 \%)$ & & & $231(36.55 \%)$ \\
\hline Gallstone number & & & 3.374 & $<0.001^{*}$ & \\
\hline 1 & $176(15.59 \%)$ & $34(9.97 \%)$ & & & $92(14.55 \%)$ \\
\hline$\geq 2$ & $953(84.41 \%)$ & $307(90.03 \%)$ & & & $540(85.44 \%)$ \\
\hline Gallstone diameter $囚 \mathrm{~mm}$ ) & & & 34.977 & $<0.001^{*}$ & \\
\hline
\end{tabular}




\begin{tabular}{|c|c|c|c|c|c|}
\hline$\triangle 3 \mathrm{~mm}$ & 197(17.45\%) & 108(31.67\%) & & & $141(22.31 \%)$ \\
\hline $3-10 \mathrm{~mm}$ & $745(65.99 \%)$ & $198(58.06 \%)$ & & & $384(60.76 \%)$ \\
\hline$₫ 10 \mathrm{~mm}$ & 187(16.56\%) & $35(10.26 \%)$ & & & $107(16.93 \%)$ \\
\hline Gallstone shape & & & 11.785 & 0.008 & \\
\hline Sphere & $623(55.18 \%)$ & $196(57.48 \%)$ & & & $364(57.59 \%)$ \\
\hline Irregular & $316(27.99 \%)$ & $70(20.53 \%)$ & & & $137(21.68 \%)$ \\
\hline Sand-like & $190(16.83 \%)$ & $75(21.99 \%)$ & & & $131(20.73 \%)$ \\
\hline Bile duct stones & & & 43.594 & $<0.001^{*}$ & \\
\hline No & $935(82.82 \%)$ & $225(65.98 \%)$ & & & $498(78.80 \%)$ \\
\hline Yes & 194(17.18\%) & $116(34.02 \%)$ & & & $134(21.20 \%)$ \\
\hline Diameter of CBD $\nabla m m \rrbracket$ & & & 0.003 & 0.958 & \\
\hline$\leq 10$ & $990(87.69 \%)$ & $298(87.39 \%)$ & & & $548(86.71 \%)$ \\
\hline$>10$ & 139(12.31\%) & $43(12.61 \%)$ & & & $84(13.29 \%)$ \\
\hline ALT囚U/L】 & & & 100.583 & $<0.001$ & \\
\hline$₫ 150 U / L$ & $959(84.94 \%)$ & $203(59.53 \%)$ & & & $514(81.33 \%)$ \\
\hline$\geq 150 \mathrm{U} / \mathrm{L}$ & $170(15.06 \%)$ & $138(40.47 \%)$ & & & $118(18.67 \%)$ \\
\hline AST『U/L】 & & & 138.204 & $<0.001$ & \\
\hline$₫ 53.6 \mathrm{U} / \mathrm{L}$ & $868(76.88 \%)$ & $147(43.11 \%)$ & & & $450(71.20 \%)$ \\
\hline$\geq 53.6 \mathrm{U} / \mathrm{L}$ & $261(23.12 \%)$ & $194(56.89 \%)$ & & & $182(28.80 \%)$ \\
\hline AST/ALT & & & 0.611 & 0.435 & \\
\hline$₫ 1.0$ & $643(56.95 \%)$ & $203(59.53 \%)$ & & & $378(59.81 \%)$ \\
\hline$\geq 1.0$ & $486(43.05 \%)$ & $138(40.47 \%)$ & & & $254(40.19 \%)$ \\
\hline GGTهU/L】 & & & 161.936 & $<0.001$ & \\
\hline$\otimes 150$ & $838(74.22 \%)$ & $125(36.66 \%)$ & & & $393(62.18 \%)$ \\
\hline$\geq 150$ & $291(25.78 \%)$ & $216(63.34 \%)$ & & & $239(37.82 \%)$ \\
\hline AKP囚U/L】 & & & 81.286 & $<0.001$ & \\
\hline$\otimes 125$ & $830(73.52 \%)$ & $161(47.21 \%)$ & & & $432(68.35 \%)$ \\
\hline$\geq 125$ & $299(26.48 \%)$ & $180(52.79 \%)$ & & & $200(31.65 \%)$ \\
\hline TBIL『mg/dL】 & & & 96.221 & $<0.001$ & \\
\hline
\end{tabular}




\begin{tabular}{|c|c|c|c|c|c|}
\hline$\nabla 1.4$ & $828(73.34 \%)$ & $152(44.57 \%)$ & & & $422(66.77 \%)$ \\
\hline$\geq 1.4$ & $301(26.66 \%)$ & 189(55.43\%) & & & $210(33.23 \%)$ \\
\hline IBIL『mg/dL】 & & & 30.791 & $<0.001$ & \\
\hline$\otimes 0.8$ & 1013(89.73\%) & $266(78.01 \%)$ & & & $540(85.44 \%)$ \\
\hline$\geq 0.8$ & $116(10.27 \%)$ & 75(21.99\%) & & & $92(14.56 \%)$ \\
\hline DBIL『mg/dL】 & & & 9.885 & 0.002 & \\
\hline$\geq 1.0$ & $28(2.48 \%)$ & $21(6.16 \%)$ & & & 19(3.01\%) \\
\hline$\otimes 1.0$ & $1101(97.52 \%)$ & $320(93.84 \%)$ & & & 613(96.99\%) \\
\hline WBCヌÍ109/L】 & & & 186.352 & $<0.001$ & \\
\hline \10 & $885(78.39 \%)$ & 134(39.30\%) & & & $444(70.25 \%)$ \\
\hline$\geq 10$ & $244(21.61 \%)$ & $207(60.70 \%)$ & & & $188(29.75 \%)$ \\
\hline GRAN\%ヌ\%】 & & & 162.649 & $<0.001$ & \\
\hline$\varangle 80$ & $823(72.90 \%)$ & 119(34.90\%) & & & $408(64.56 \%)$ \\
\hline$\geq 80$ & $306(27.10 \%)$ & $222(65.10 \%)$ & & & $224(35.44 \%)$ \\
\hline
\end{tabular}

Table Note: $P<0.05$ was statistically significant.

AKP, Alkaline phosphatase $₫ A L T$, Alanine transaminase; AST, Aspartate transaminase; CBD, Common bile duct; DBIL, Direct bilirubin; GGT, Gamma-glutamyl transferase; GRAN\%, Granulocyte\%; IBIL, Indirect bilirubin; TBIL, Total bilirubin; WBC, White blood cell count

Table-2. The results of affecting ABP induction by multivariate logistic stepwise regression analysis in training set 


\begin{tabular}{|c|c|c|c|c|c|}
\hline Variable & B & SE & Wald $\chi^{2}$ & $\mathbf{P}$ & OR (95\%Cl) \\
\hline Constant quantity & -1.586 & 0.406 & 15.301 & $<0.001$ & $0.205(0.091,0.449)$ \\
\hline Male & 0.662 & 0.154 & 18.479 & $<0.001$ & $1.940(1.436,2.628)$ \\
\hline Diabetes history & 0.824 & 0.162 & 25.770 & $<0.001$ & $2.279(1.657,3.132)$ \\
\hline 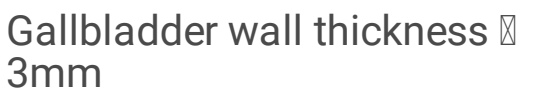 & -0.457 & 0.171 & 7.178 & 0.007 & $0.633(0.452,0.882)$ \\
\hline $\begin{array}{l}\text { Gallstone diameter } \\
\text { (vs. } ₫ 3 \mathrm{~mm} \rrbracket\end{array}$ & -- & -- & -- & -- & -- \\
\hline $3-10 \mathrm{~mm}$ & -0.716 & 0.181 & 15.641 & $<0.001$ & $0.489(0.343,0.697)$ \\
\hline$\otimes 10 \mathrm{~mm}$ & -0.996 & 0.263 & 14.352 & $<0.001$ & $0.369(0.218,0.614)$ \\
\hline Bile duct stones & 0.724 & 0.210 & 11.861 & 0.001 & $2.062(1.366,3.115)$ \\
\hline Diameter of CBD $₫ 10 \mathrm{~mm}$ & -0.679 & 0.270 & 6.335 & 0.012 & $0.507(0.296,0.853)$ \\
\hline AST $\otimes 53.6 \mathrm{U} / \mathrm{L}$ & 0.700 & 0.199 & 12.360 & $<0.001$ & $2.013(1.362,2.974)$ \\
\hline GGTه150U/L & 0.836 & 0.195 & 18.416 & $<0.001$ & $2.306(1.574,3.380)$ \\
\hline DBIL $\otimes 1.0 \mathrm{mg} / \mathrm{dL}$ & -1.334 & 0.372 & 12.871 & $<0.001$ & $0.263(0.127,0.549)$ \\
\hline WBC凶10Í $10^{9}$ & 1.435 & 0.16 & 80.093 & $<0.001$ & $4.201(3.073,5.767)$ \\
\hline GRAN\% $₫ 80 \%$ & 1.098 & 0.156 & 49.788 & $<0.001$ & $2.997(2.211,4.071)$ \\
\hline
\end{tabular}

Table Note: $P<0.05$ was statistically significant.

AST, Aspartate transaminase; CBD, Common bile duct; DBIL, Direct bilirubin; GGT, Gamma-glutamyl transferase; GRAN\%, Granulocyte\%; WBC, White blood cell count.

\section{Figures}




\section{Case data of gallstone patients were}

collected in recent 10 years

\section{Inclusion criteria :}

Gallstone patients

diagnosed by imaging

examination like

ultrasound, CT and

MRCP

\section{Exclusion criteria:}

(1)Incomplete data

(2)Non-biliary pancreatitis

(3)Ambiguous diagnosis

(4)With other major diseases

(5) Pregnancy and lactation
ABP diagnostic criteria:

(1) Gallstones confirmed by imaging examination

(2) Clinical signs

(3)Laboratory test

(4) Exclusion of other causes
Eligible case data (2102 cases) were grouped randomly in a ratio of 7:3
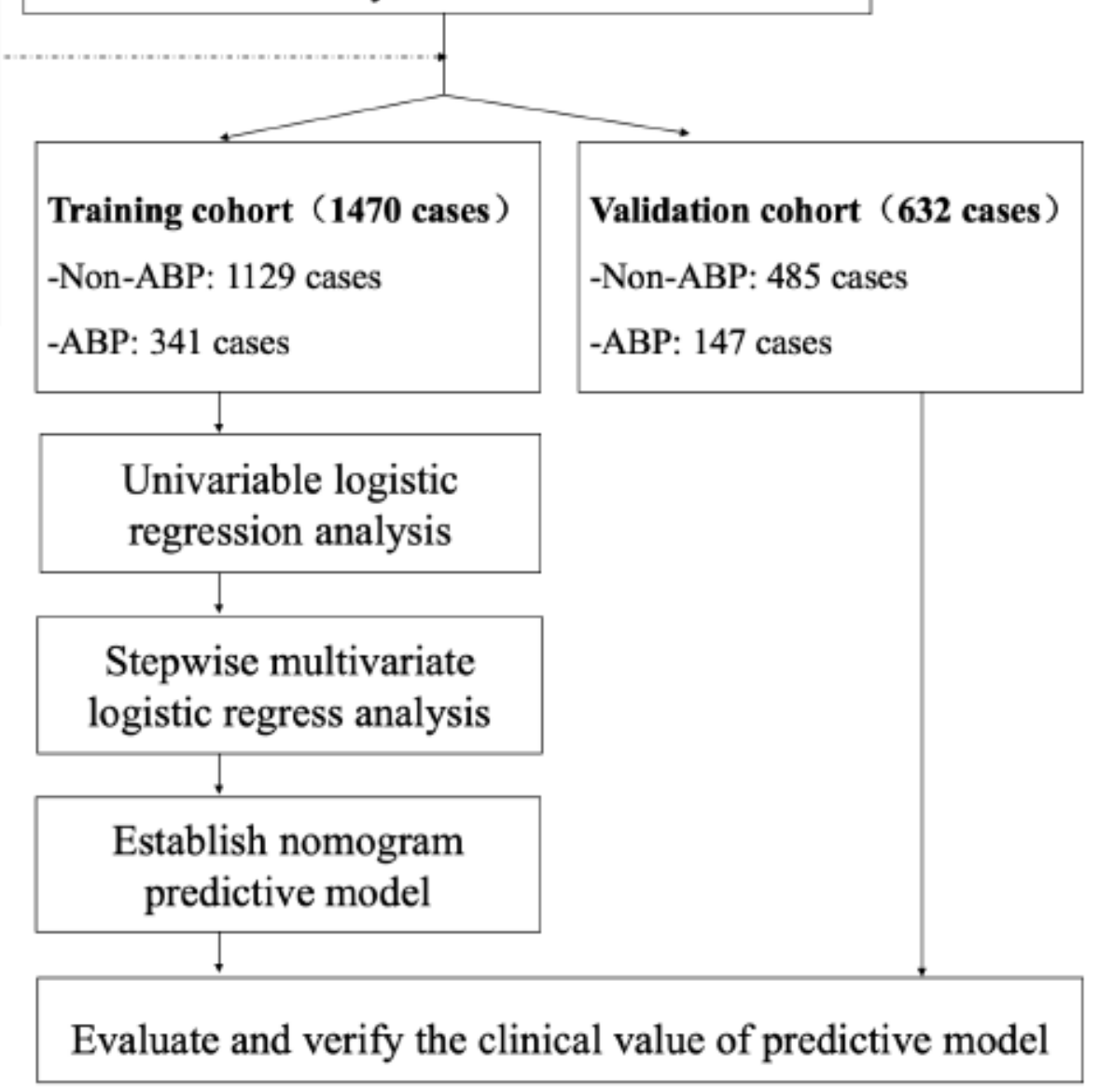

Figure 1

Flow chart of the study 


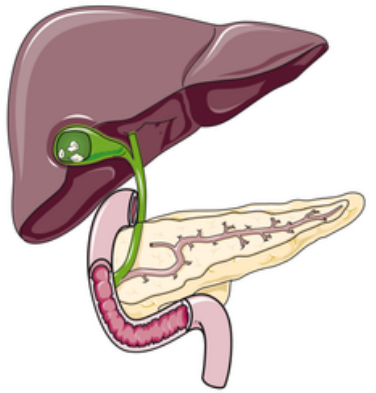

Gallstones
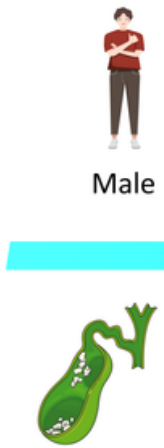

Gallstone diameter $\leq 3 \mathrm{~mm}$ Gallbladder wall thickness $<3 \mathrm{~mm}$

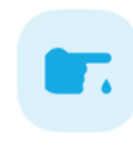

Diabetes
Coexisting CBD stones $\mathrm{CBD}$ diameter $\leq 10 \mathrm{~mm}$

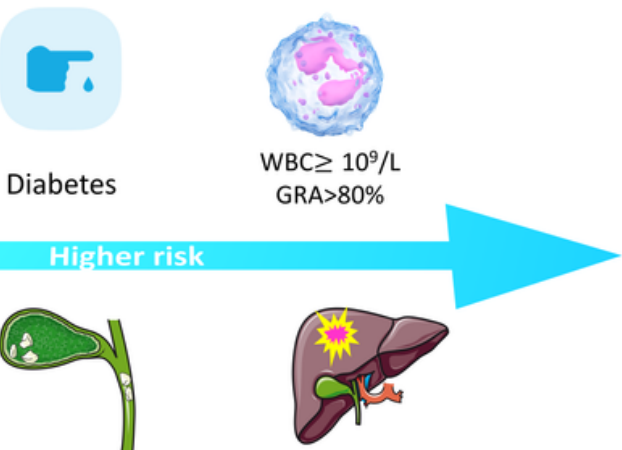

WBC $\geq 10^{9} / \mathrm{L}$

GRA $>80 \%$

AST $\geq 53.6 \mathrm{U} / \mathrm{L}$ $\mathrm{GGT} \geq 150 \mathrm{U} / \mathrm{L}$ $\mathrm{DBIL} \geq 17.1 \mathrm{mg} / \mathrm{dL}$

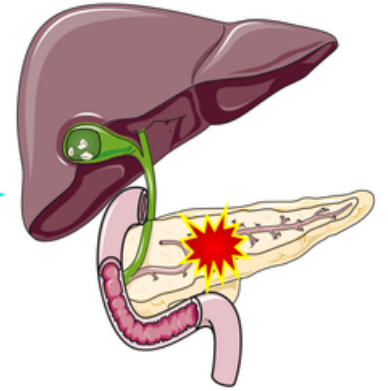

Acute biliary pancreatitis

Independent risk factors for $\mathrm{ABP}$ in patients with gallstones

\section{Figure 2}

Independent risk factors for ABP in patients with gallstones 
Points

$\begin{array}{lllllllllll}0 & 10 & 20 & 30 & 40 & 50 & 60 & 70 & 80 & 90 & 100\end{array}$

Sex

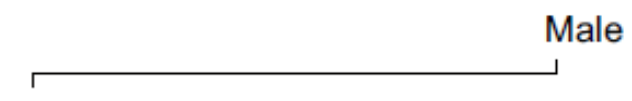

\section{Female}

Diabetes history

Gallbladder wall

thickness, $\mathrm{mm}$

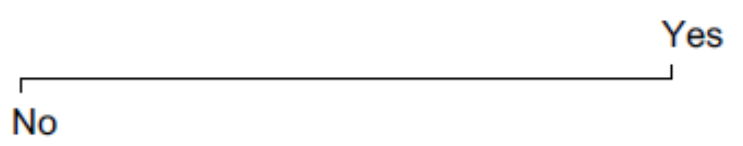

Gallstone diameter, $\mathrm{mm} \underset{>10}{\stackrel{3 \sim 10}{\sim}<3}<$

Coexisting CBD stone

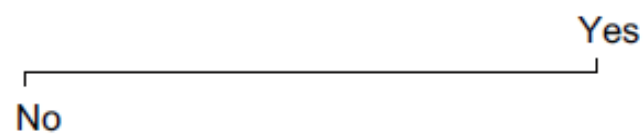

CBD diameter, $\mathrm{mm}$

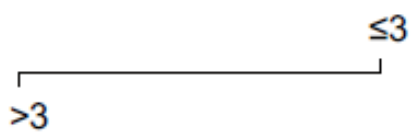

$\leq 10$

$$
>10
$$

AST, U/L

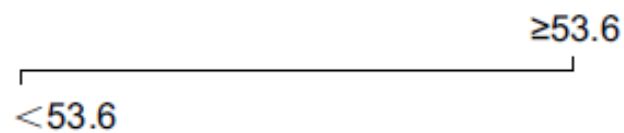

GGT, U/L

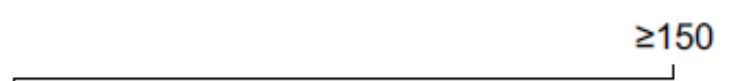

DBIL, $\mathrm{mg} / \mathrm{dL}$

$$
<150
$$

WBC, $\times 10^{9} / \mathrm{L}$

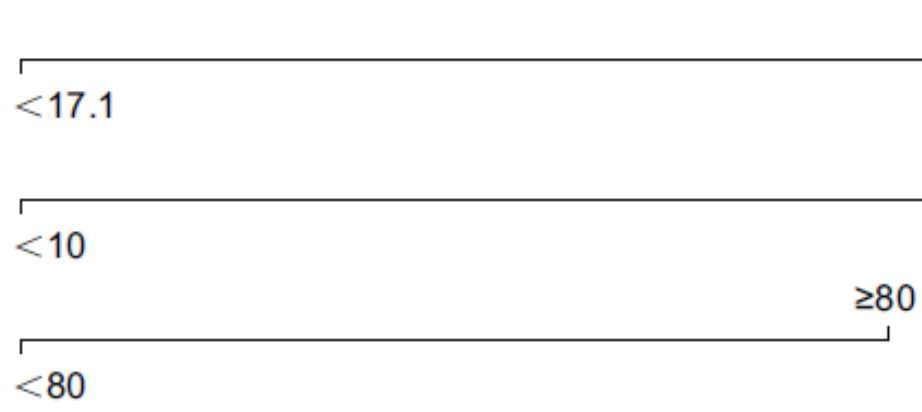

Total points

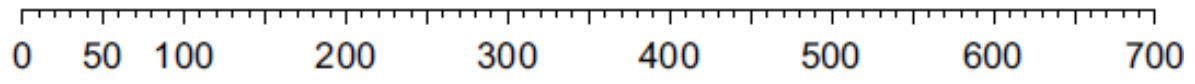

ABP incidence risk

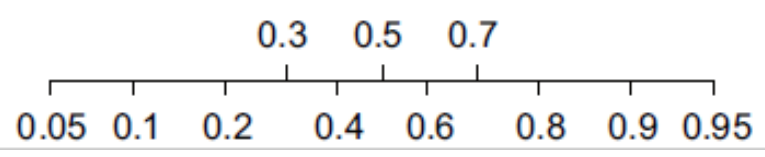

Figure 3

The nomogram to estimate the risk of ABP presence in patients with gallstones 


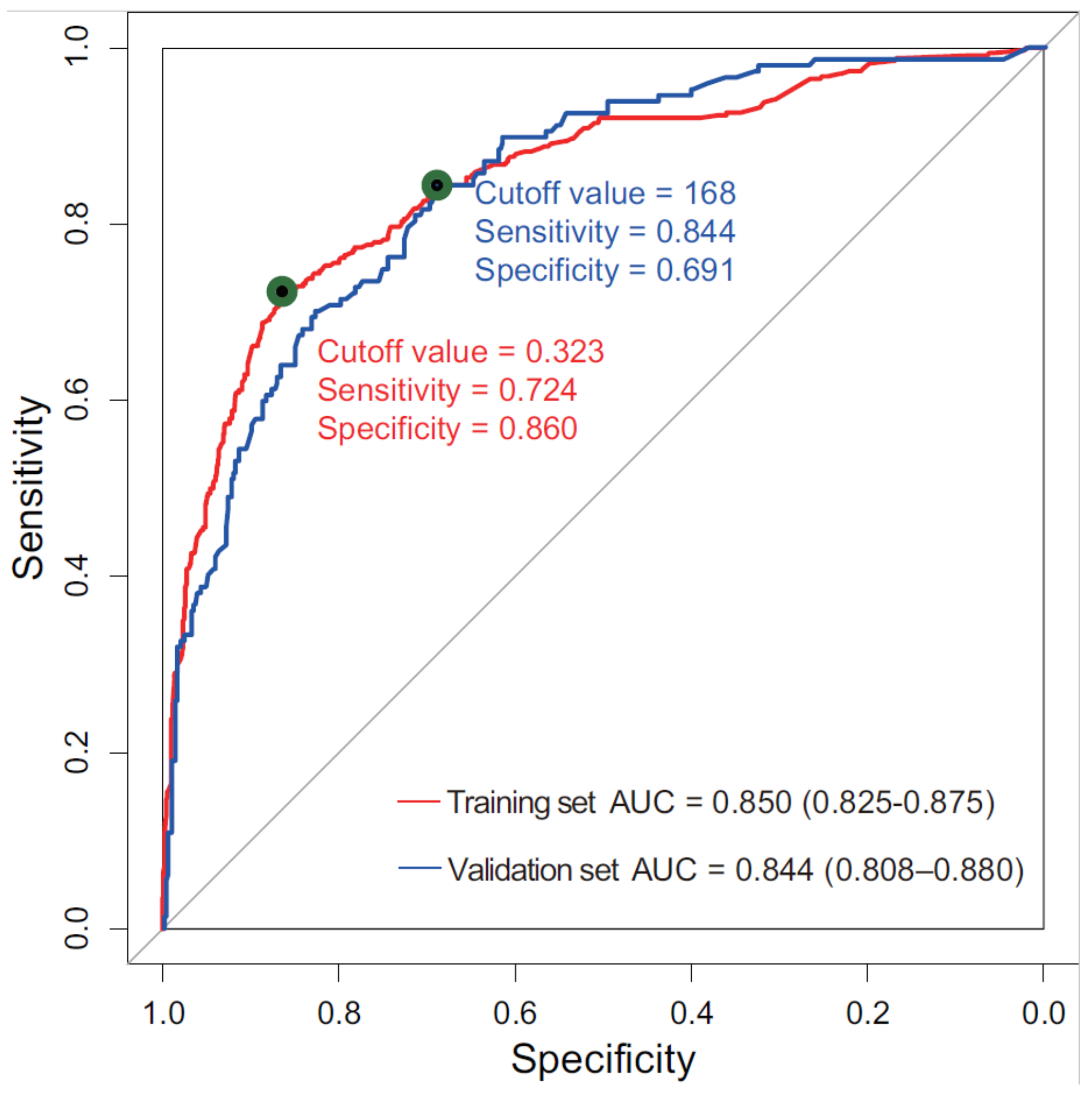

Figure 4

The AUC of the model from observed data (nomogram) was 0.850 in training set and 0.844 in validation set respectively. In training set, the cut-off value was 0.323 with a sensitivity of 0.724 and a specificity of 0.860 . In validation set, the cut-off value was 0.168 with a sensitivity of 0.844 and a specificity of 0.691 . AUC=area under ROC 


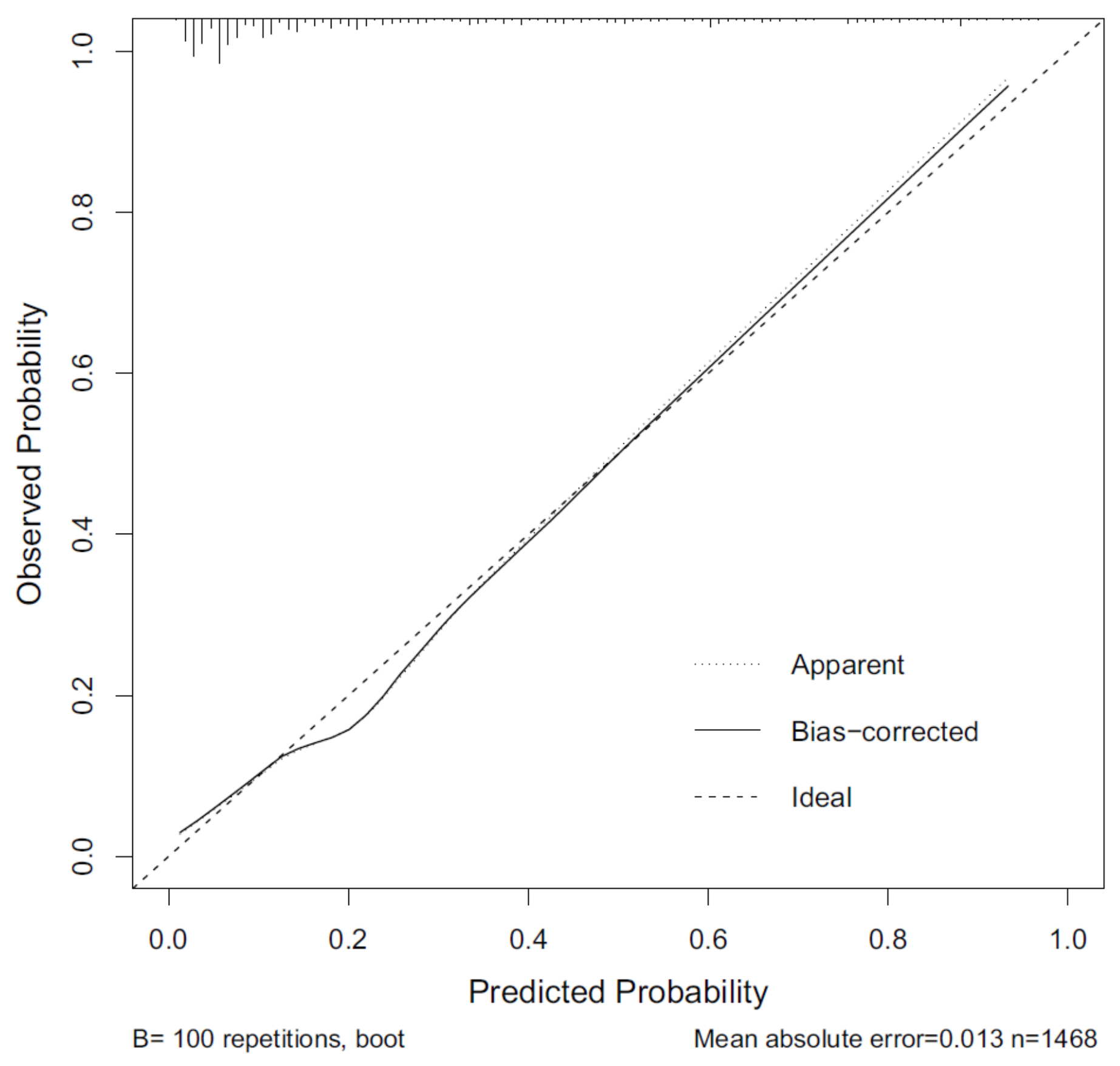

\section{Figure 5}

The decision curve analysis (DCA) curve for the nomogram. The Y-axis shows the net benefit. The X-axis shows the corresponding risk threshold. The thin line represents the assumption that all patients have acute biliary pancreatitis (ABP). The thick line represents the assumption that no patients have ABP. The red line represents the nomogram. The decision curve in the validation cohort indicated that if the threshold probability is between 0.1 and 1.0 , then using the nomogram to predict ABP adds more benefit than the treat-all-patients scheme or treat-none scheme 


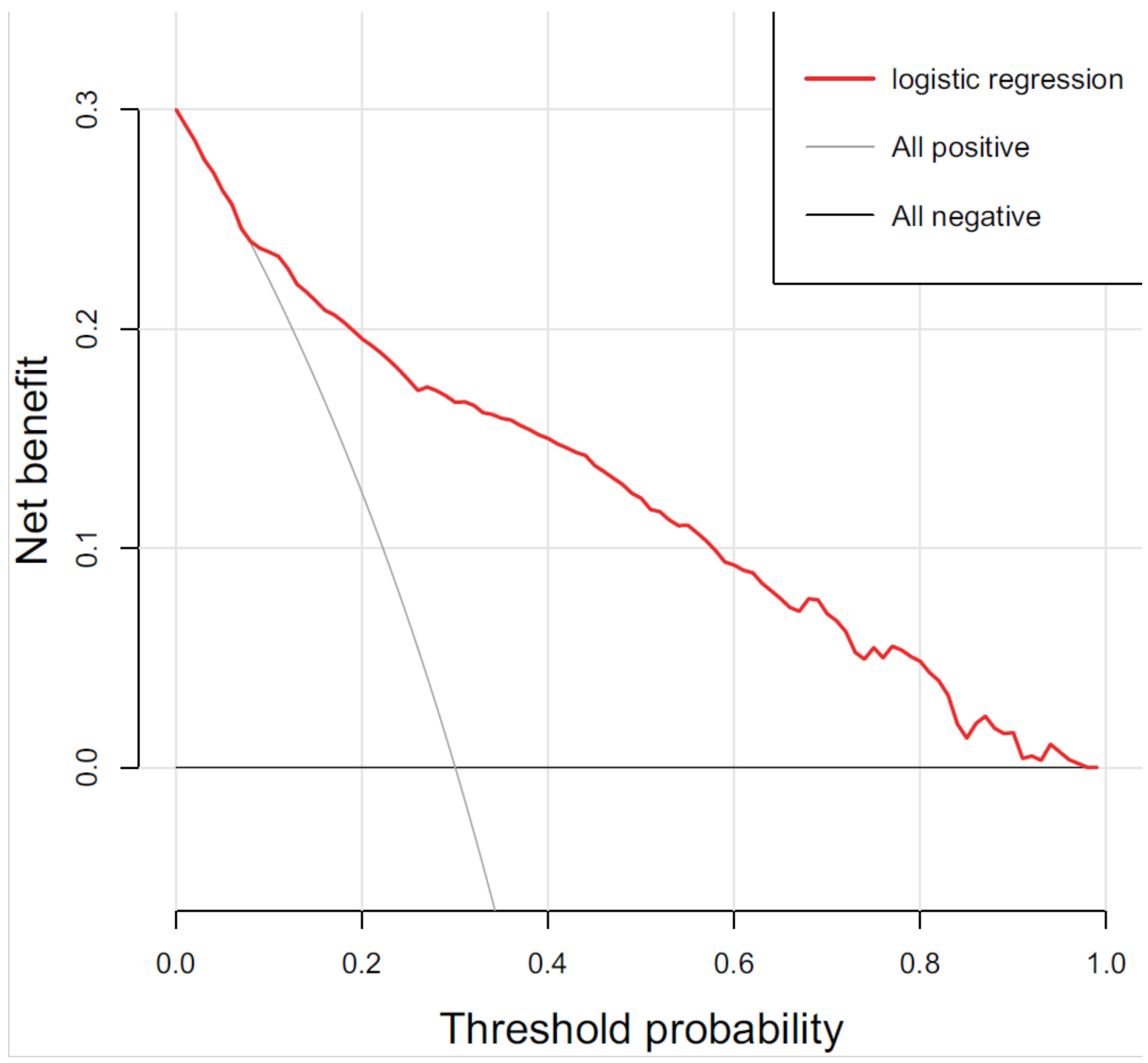

Figure 6

The decision curve analysis (DCA) curve for the nomogram. The Y-axis shows the net benefit. The X-axis shows the corresponding risk threshold. The thin line represents the assumption that all patients have acute biliary pancreatitis (ABP). The thick line represents the assumption that no patients have ABP. The red line represents the nomogram. The decision curve in the validation cohort indicated that if the threshold probability is between 0.1 and 1.0, then using the nomogram to predict ABP adds more benefit than the treat-all-patients scheme or treat-none scheme

\section{Supplementary Files}


This is a list of supplementary files associated with this preprint. Click to download.

- STROBEchecklistv4combinedPlosMedicine.docx 